\title{
Utilization of sweet potato starch wastewater for biofertilizer production by Bacillus amyloliquefaciens
}

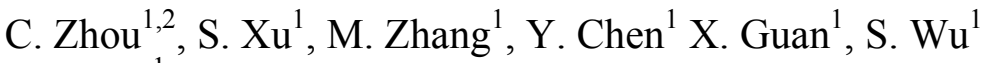 \\ \& $\mathrm{Z} \mathrm{Bai}^{1}$ \\ ${ }^{1}$ Research Center for Eco-Environmental Sciences, \\ Chinese Academy of Sciences, China \\ ${ }^{2}$ Tianjin University of Technology, China
}

\begin{abstract}
Effluents from the sweet potato starch industry possess a high load of Chemical Oxygen Demand (COD) (10-35 g/l). The treatment of such wastewater by conventional activated sludge processes consumes a lot of energy, resulting in high treatment costs. A plant growth promoting bacterium (PGPB) Bacillus amyloliquefaciens strain EBL11 was isolated from soil. The isolate EBL11 performed a good capacity of promoting tea plant growth, which indicated that the strain can be used as a nonchemical alternative biofertilizer. In this study, we investigated the carbon and nutrient rich industrial processing effluents of sweet potato wastewater (SPW) as substrate for the cultivation of the biofertilizer strain EBL11. The isolate EBL11 can grow well using SPW as the culture media. The maximum yield of $8.7 \times 10^{9} \mathrm{CFU} / \mathrm{ml}$ of the isolate EBL11 was reached in $18 \mathrm{~h}$ at $32^{\circ} \mathrm{C}$, pH 7.0 using the SPW as substrate. In conclusion, it is feasible to develop a hybrid biotechnological process, integrating the production of environmental friendly biofertilizer with treatment of intractable wastewater.

Keywords: Bacillus amyloliquefaciens, biofertilizer, plant growth promoting bacteria, sweet potato starch wastewater, tea.
\end{abstract}

\section{Introduction}

Wastewater from the sweet potato starch industry contains a high load of protein, pectin, and starchy materials with the COD of 10,000-35,000 mg/l [1]. Due to 
the high loading of COD, the treatment of such wastewater by conventional activated sludge processes can be complicated and costly consuming a lot of energy, resulting in high treatment costs. However, the wastewater with a high concentration of nutrients might be a good culture medium for microorganism cultivation.

Bacillus amyloliquefaciens was originally described as a potent producer of liquefying amylase and other industrial ectoenzymes [2]. Many strains of B. amyloliquefaciens as plant growth-promoting bacteria (PGPB) were isolated from soil, associated with plant roots and exert beneficial effects on plant development $[3,4]$. In the present work, a strain of B. amyloliquefaciens EBL11 was isolated from soil, which could improve the growth of tea plant and tea yield. Therefore, the plant growth promoting effect of this strain suggests a potential application as biological fertilizer.

In this study, we investigated a feasibility of production of biofertilizer by $B$. amyloliquefaciens EBL11 using the sweet potato wastewater (SPW).

\section{Materials and methods}

\subsection{Microorganism}

An anti-fungi bacterial strain EBL11 was isolated on Potato-dextrose Agar (PDA) medium in Petri dish from soil, and maintained on the PDA slant tubes.

\subsection{Optimization of culture conditions}

SPW samples were collected from a sweet potato starch process waste stream in Changsha, China, mainly containing COD $17000 \mathrm{mg} / \mathrm{l}$. The $\mathrm{pH}$ of SPW was adjusted to 7.0 by $\mathrm{NaOH}$ when the SPW was used as the culture medium. The starch, pectin and sugars in the SPW were used as the main carbon source throughout the investigation.

The isolate EBL11 was grown on the PDA plates at $30^{\circ} \mathrm{C}$ for 2 days. A single clone was inoculated into $250-\mathrm{ml}$ flask containing $100 \mathrm{ml}$ of the PDA medium at $30^{\circ} \mathrm{C}$ for $24 \mathrm{~h}$. This seed culture was used to initiate the growth in fermentation medium used in this study. The basic medium (BM) is composed of as follows (g/l): $\left(\mathrm{NH}_{4}\right)_{2} \mathrm{SO}_{4} 5, \mathrm{MgSO}_{4} \cdot 7 \mathrm{H}_{2} \mathrm{O} 0.10, \mathrm{KH}_{2} \mathrm{PO}_{4} 0.50, \mathrm{NaCl} 0.50$, and $\mathrm{K}_{2} \mathrm{HPO}_{4}$ 1.50. The fermentation was conducted in $250 \mathrm{ml}$ Erlenmeyer flasks containing $100 \mathrm{ml}$ medium inoculated with $5 \mathrm{ml}$ of seed culture. Unless otherwise stated, the agitation rate and incubation temperature were $200 \mathrm{r} / \mathrm{min}$ and $30^{\circ} \mathrm{C}$, respectively.

All experiments were conducted in duplicate and the average values are reported. Key results were repeated three times to establish their validity.

\subsection{Tea plant growth promotion}

Tea plants (C. sinensis) from tea plantation (3 years old) of semi-tropical uplands, Hunan, China $\left(113^{\circ} 19^{\prime} \mathrm{E}, 28^{\circ} 33^{\prime} \mathrm{N}\right)$ were used to study the efficacy of the PGPB strain EBL11 on the yield of tea. The plantation belongs to the typical 
red soil. Tea plants have no clear differences owing to unified management conditions.

The treatments were conducted by irrigation of the biofertilizer (fermented SPW by strain EBL11) in four levels: without biofertilizer (CK), $100 \mathrm{ml} / \mathrm{m}^{2}$, $200 \mathrm{ml} / \mathrm{m}^{2}$ and $300 \mathrm{ml} / \mathrm{m}^{2}$ for three times monthly. There were three replications with each replicate consisting of 50 bushes. The experiment was laid out in a randomized block design with a plot size of $10 \mathrm{~m} \times 20 \mathrm{~m}$. Tea production was measured by one-hundred-bud weight and then fresh tea leaves was treated including fixation, rolling and baking, to prepare the green tea by the same procedure [5].

\section{Results and discussion}

\subsection{Effect of carbon sources on the isolate EBL11 growth}

The fermentation medium contained $100 \mathrm{ml} \mathrm{BM}$, and 2\% each carbon sources, including sugar, D-glucose, soluble starch, and SPW (90 ml) with BM (10 ml). After inoculation with $5 \mathrm{ml}$ of seed culture, the medium was incubated at $30^{\circ} \mathrm{C}$ for $20 \mathrm{~h}$. The effect of carbon sources on the production of the isolate EBL11 are presented in Fig. 1. It was found that SPW was a suitable carbon source for the isolate EBL11 cultivation.

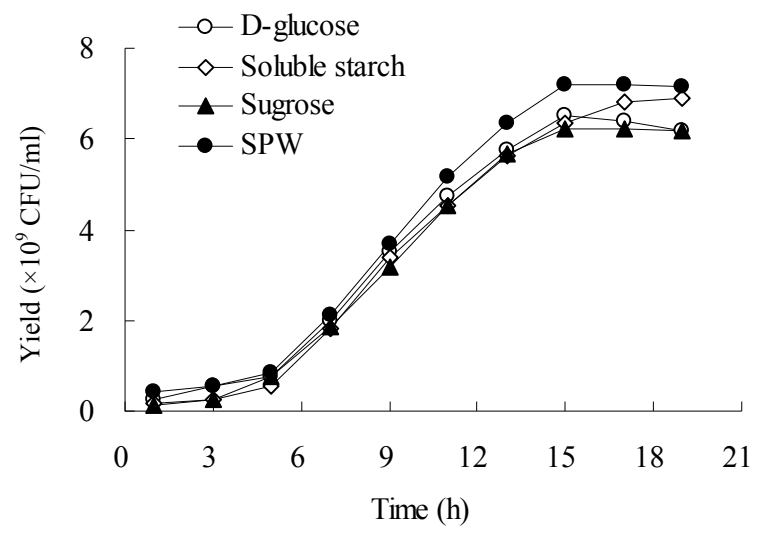

Figure 1: Effect of different carbon sources on the isolate EBL11 growth.

\subsection{Effect of nitrogen sources on the isolate EBL11 growth}

The fermentation medium contained $90 \mathrm{ml} \mathrm{SPW}$ as carbon source, $10 \mathrm{ml} \mathrm{BM}$, and $0.5 \%$ different nitrogen sources, including corn steep liquor, potassium nitrate, ammonium sulfate. A control experiment was conducted without addition of nitrogen source. After inoculation with $5 \mathrm{ml}$ of inoculum, the medium was incubated at $30^{\circ} \mathrm{C}$ for $20 \mathrm{~h}$. The results of impact of nitrogen sources on the production of the isolate EBL11 are presented in Fig. 2. It was found that corn steep liquor was the most efficient nitrogen source for production of the strain. 


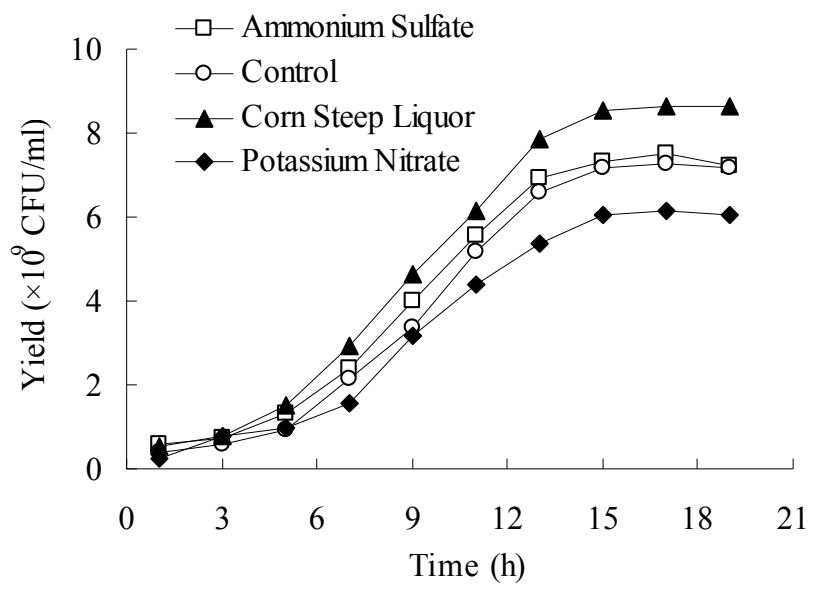

Figure 2: $\quad$ Effect of different nitrogen sources on the isolate EBL11 growth.

\subsection{Effect of culture temperature on the isolate EBL11 production}

The fermentation medium contained $100 \mathrm{ml}$ SPW with $0.3 \%$ of corn steep liquor. After inoculation with $5 \mathrm{ml}$ of inoculum, the medium was incubated at $24,28,32,36$ and $40^{\circ} \mathrm{C}$, respectively. The time courses of the isolate EBL11 production at different temperature are shown in Fig. 3. The maximum yield of $8.7 \times 10^{9} \mathrm{CFU} / \mathrm{ml}$ was reached at $32^{\circ} \mathrm{C}$ after $18 \mathrm{~h}$ fermentation.

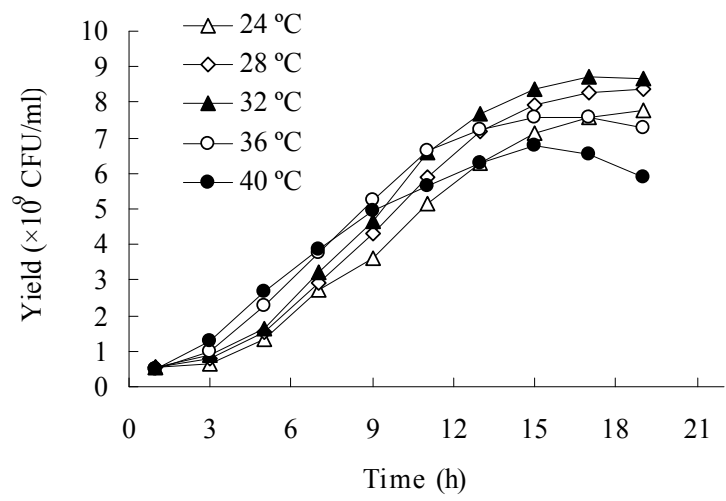

Figure 3: Effect of culture temperature on the isolate EBL11 growth.

\subsection{Isolate EBL11 promoting the leaves growth of tea plants}

B. amyloliquefaciens EBL11 was investigated for possible beneficial effects on the growth of tea plants. The results revealed that application of the biofertilizer was useful for the promotion of tea growth (Fig. 4). The tea yield was increased 
significantly by irrigating $200 \mathrm{ml} / \mathrm{m}^{2}$ and $300 \mathrm{ml} / \mathrm{m}^{2}$ of fermented SPW by the isolate EBL11. In general, the results confirm those of previously published studies that have investigated the growth response of plants to inoculation with B. amyloliquefaciens $[6,7]$. B. amyloliquefaciens was discovered to be soilborne and plant associated, i.e., to possess the abilities to stimulate plant growth and suppress plant pathogens by promoting seedling emergence, plant biomass, and disease control [2].

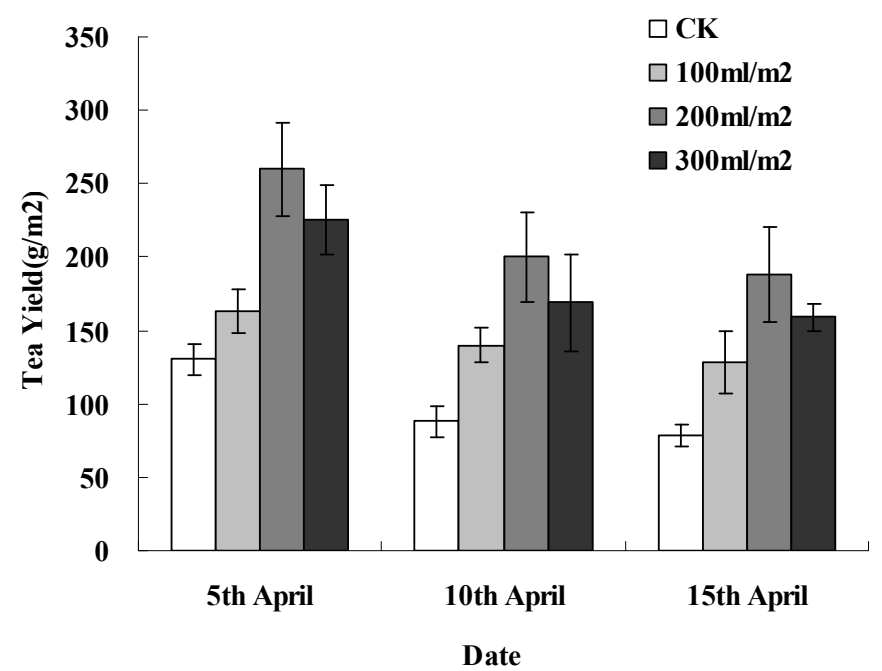

Figure 4: $\quad$ Effect of biofertilizer application on the yield of tea.

SPW is one of the most intractable wastewater because of its high strength of COD. A few studies have already focused on the utilization of such high organic loading wastewater as substrates or production media in fermentation processes $[1,8]$. As far as the authors are aware, no research case was found in the literature for utilization of SPW for biofertilizer of $B$. amyloliquefaciens production.

\section{Conclusions}

A newly isolated B. amyloliquefaciens EBL11 from soil can be used as a nonchemical alternative biofertilizer for promoting tea plant growth and increase tea yield. It is feasible to develop a hybrid biotechnological process, integrating the production of environmental friendly biofertilizer with treatment of intractable SPW. 


\section{Acknowledgements}

This work was supported by the Key Program of Chinese Academy of Sciences (No. KZZD-EW-09-3), the Beijing Science and Technology Program (No. D101105046410004) and the Tianjin Hi-tech Support Program Key Projects (No. 11ZCGYSF01400). We thank Wuxi Zhongkehuoli Biotechnology Co. Ltd for the pilot scale biofertilizer production.

\section{References}

[1] Bai, Z., Gu, L., Su, Y., et al., Utilization of sweet potato starch wastewater and monosodium glutamate wastewater for cultivation of an anti-fungal biocontrol agent Paenibacillus polymyxa (Chapter 13). Fungicides, ed. O. Carisse, InTech Open Access Publisher: Rijeka, Croatia, pp. 261-270, 2010.

[2] Geng, W., Cao, M., Song, C, et al., Complete genome sequence of Bacillus amyloliquefaciens LL3, which exhibits glutamic acid-independent production of poly- $\gamma$-glutamic acid. Journal of Bacteriology, 193(13), pp. 3393-3394, 2011.

[3] Chen, X.H., Koumoutsi, A., Scholz, R., et al., Comparative analysis of the complete genome sequence of the plant growth-promoting bacterium Bacillus amyloliquefaciens FZB42. Nature Biotechnology, 25(9), pp. 10071014, 2007.

[4] Hu, H.Q, Li, X.S. and He, H., Characterization of an antimicrobial material from a newly isolated Bacillus amyloliquefaciens from mangrove for biocontrol of Capsicum bacterial wilt. Biological Control, 54(3), pp. 359365, 2010.

[5] The First Research Institute of China Standards Publisher, Standards collection for tea, China Standards Publisher: Beijing, PR China, 2003.

[6] Adesemoye, A.O., Torbert, H.A. and Kloepper, J.W., Increased plant uptake of nitrogen from ${ }^{15} \mathrm{~N}$-depleted fertilizer using plant growth promoting rhizobacteria. Applied Soil Ecology, 46(1): pp. 54-58, 2010.

[7] Herman, M.A.B., Nault, B.A. and Smart, C.D., Effects of plant growthpromoting rhizobacteria on bell pepper production and green peach aphid infestations in New York. Crop Protection, 27(6), pp. 996-1002, 2008.

[8] Huang, L.P., Jin, B., Lant, P., et al., Direct fermentation of potato starch wastewater to lactic acid by Rhizopus oryzae and Rhizopus arrhizus. Bioprocess and Biosystems Engineering, 27(4), pp. 229-238, 2005. 